um die Durchführung einer Agrarpolitik ging, deren Ziel die Produktivitäts-Steigerung war. Dabei wurden brutale, abstoßende Mittel eingesetzt [...]. Die Steigerung der Produktivität ist vielleicht an sich kein guter Zweck, aber sicher auch kein schlechter. Jedenfalls ist sie rational. Und die Kulaken wurden im Zusammenhang mit der Durchsetzung dieses Ziels ausgehungert, nicht, weil es wünschenswert schien, sie als solche auszurotten. Man kann nun richtigerweise sagen, daß es für einen toten Kulaken oder einen toten Juden kaum einen Unterschied macht, welche Motive seine Henker hatten. Aber für die Henker - und für unsere Einschätzung ihrer Taten - macht es einen großen Unterschied. ${ }^{152}$

\title{
3.2 Die Totalitarismusstudien von Franz Borkenau und James Burnham
}

Auch wenn von einer zumindest indirekten Einflussnahme von Macdonald aufArendts Reflexionen zur »ökonomischen Zwecklosigkeit« der nationalsozialistischen Judenvernichtung ausgegangen werden kann, fielen gleichwohl seine Ausführungen zum singulären Charakter des Holocaust und der Vergleich der nationalsozialistischen mit den stalinistischen Massenverbrechen fortan in der Geschichte der Totalitarismustheorie dem Vergessen anheim. Im Unterschied dazu nimmt, wie gezeigt, die 1940 veröffentlichte Untersuchung The Totalitarian Enemy des früheren Parteikommunisten Borkenau einen zentralen Platz ein. ${ }^{153}$ Allerdings stand noch in den frühen 1930er-Jahren bei Borkenau eine explizit marxistische Auffassung am Anfang der Auseinandersetzung mit einem neuartigen Herrschaftsphänomen, für das er später den Begriff des Totalitarismus wählte. Denn noch in seinem 1933 veröffentlichten Aufsatz Zur Soziologie des Faschismus rubrizierte er trotz aller Unterschiede den Nationalsozialismus und den italienischen Faschismus unter den Sammelbegriff»Faschismus«. Borkenaus Einschätzung zufolge war der Faschismus eine »exklusive Diktatur einer totalitären Partei«, der ausschließlich unter den verschärften antagonistischen Bedingungen der kapitalistischen liberalen Demokratien an die Macht gelangen konnte. ${ }^{154}$

Die 1936 veröffentlichte Studie über Pareto nahm auf dem Weg zum borkenauschen Totalitarismusbegriff gewissermaßen eine Mittelstellung ein. Obwohl Borkenau sich vom Faschismusbegriff zur Charakterisierung der Gemeinsamkeiten zwischen Bolschewismus, Faschismus und Nationalsozialismus inzwischen gelöst hatte, suchte er noch nach einer identifizierenden Kategorie für die neuartigen Diktaturen. Gleichwohl erkannte er bereits in allen drei Herrschaftssystemen ähnliche Merkmale. Denn nach Borkenaus Auffassung herrschte nämlich in den drei Diktaturen eine neue Elite, d.h. eine Partei und ein Führer, die den gesamten politischen und wirtschaftlichen Bereich kontrollieren und imstande waren, über die gesellschaftlichen Klassen hinweg, durch ihre Entschlossenheit, durch den institutionalisierten Terror

152 Zit. n. ebd., S. 291 (Anm. 25).

153 Siehe hierzu das Einleitungskapitel.

154 Franz Borkenau, Zur Soziologie des Faschismus. In: Archiv für Sozialwissenschaft und Sozialpolitik, Bd. 68 (1933), H. 5, S. 513-547. Hier nach dem Wiederabdruck bei Ernst Nolte (Hg.), Theorien über den Faschismus, Köln/Berlin 1967, S. 156-181, hier S. 178. 
und durch eine pseudoreligiöse Ideologie eine innere Homogenität, d. h. eine nationale Homogenität, zu erreichen. ${ }^{155}$

Nachdem sich in den späten 1930er-Jahren der vergleichende Totalitarismusbegriff zur Kategorisierung des Nationalsozialismus, des italienischen Faschismus und des Sowjetkommunismus etabliert hatte und der komparative Ansatz zur Analyse des Totalitarismus in der Forschung allmählich Verwendung fand, erschien 1940 The Totalitarian Enemy von Borkenau. ${ }^{156}$ Das Hauptwerk Borkenaus über die totalitären Herrschaftssysteme enthielt analytische Einsichten, die sich in den späteren »klassischen« Werken zur Totalitarismustheorie von Sigmund Neumann, Hannah Arendt ${ }^{157}$ und Carl Joachim Friedrich wiederfinden lassen. ${ }^{158}$ Zudem hinterließ das totalitarismuskritische Schlüsselwerk auch beim Renegaten George Orwell Spuren. ${ }^{159}$ Borkenau verdankte im Hinblick auf die Analyse des Nationalsozialismus wesentliche Erkenntnisse unter anderem Hermann Rauschning, der zu diesem Zeitpunkt mit Die Revolution des Nihilismus ${ }^{160}$ und Gespräche mit Hitler ${ }^{161}$ zentrale Studien zum Wesen des deutschen Totalitarismus veröffentlichte. ${ }^{162}$

155 Franz Borkenau, Pareto, London 1936. Vgl. William Jones, The Path from Weimar Communism to the Cold War. Franz Borkenau and »The Totalitarian Enemy«, in: Söllner/Walkenhaus/Wieland, Totalitarismus, S. 39-43.

156 Borkenau, The Totalitarian Enemy.

157 François Furet sieht beispielsweise im »moralischen Nihilismus « und in der »Rolle der Auflösung der Klassen« analytische Parallelen zwischen Franz Borkenau und Hannah Arendt (Das Ende einer Illusion, S. 702, Anm. 63).

158 Die nach Borkenaus endgültigen Bruch mit dem Kommunismus infolge der Erfahrungen im Spanischen Bürgerkrieg entstandenen Bücher Austria and After (London 1938) sowie The New German Empire (Harmondsworth 1939) gehörten bereits zu den Veröffentlichungen, in denen der Autor eine antitotalitäre Perspektive einnahm und als Vorstudien zu seinem totalitarismustheoretischen Hauptwerk aufgefasst werden können.

159 Nachdem Ceorge Orwell bereits Spanish Cockpit und The Communist International in zwei Rezensionen als bedeutende Bücher gewürdigt hatte, besprach er auch die Totalitarismusstudie von Franz Borkenau. In der englischen Zeitung Time and Tide vom 4. Mai 1940 schrieb er: „Der Nationalsozialismus ist eine Form des Sozialismus, er ist ausdrücklich revolutionär, er unterdrückt die Privateigentümer ebenso gewiß wie die Arbeiter. Die zwei Regimes, die von verschiedenen Seiten begonnen haben, entwickeln sich schnell auf das gleiche System hin - zu einer Art oligarchischen Kollektivismus. Und gegenwärtig, wie Dr. Borkenau zeigt, entwickelt sich Deutschland in Richtung auf Rußland hin, nicht umgekehrt.« (Zit. n. Howald, George Orwell, S. 124) - Vgl. auch Bernhard Crick, George Orwell. Ein Leben, Frankfurt a. M. 1984, S. 456.

160 Hermann Rauschning, Die Revolution des Nihilismus. Kulisse und Wirklichkeit im Dritten Reich, Zürich/New York 1938.

161 Ders., Gespräche mit Hitler, Zürich 1940.

162 Im Vorwort seiner Untersuchung wies Borkenau darauf hin, dass er sich bei seinen Ausführungen zum Nationalsozialismus neben Hermann Rauschning noch auf folgende Autoren gestützt hat: Paul Sering (d. i. Richard Löwenthal), auf den Wirtschaftspublizisten Thomas Balogh sowie auf den englischen Journalisten Frederick A. Voigt. Voigt, Kommentator und führender außenpolitischer Korrespondent des Manchester Guardian, hatte einen erheblichen Anteil an der Verbreitung der vergleichenden Analyse des Nationalsozialismus und des Sowjetkommunismus in den 1930er- und 1940er-Jahren in Großbritannien. Markus Huttner (Totalitarismus und säkulare Religionen. Zur Frühgeschichte totalitarismuskritischer Begriffs- und Theoriebildung in Croßbritannien, Bonn 1999) schreibt auf S. 28 über die Bedeutung der Veröffentlichungen Voigts: „Seine Beiträge verdienen vor allem unter dem Aspekt Beachtung, als sie auf der Schnittstelle unterschiedlicher Deutungsversu- 
Die unter dem Eindruck des deutsch-sowjetischen Nichtangriffspaktes und nach dem Einmarsch der Wehrmacht und der Roten Armee in Polen im September 1939 erstellte Studie The Totalitarian Enemy enthielt als zentrale »Botschaft« die These von der Wesensverwandtschaft des »Roten Faschismus « und des »Braunen Bolschewismus «. ${ }^{163}$ Sie wurde von dem ehemaligen Mitarbeiter des Frankfurter Institutes für Sozialforschung aufgrund der für die westlichen Staaten politisch extrem zugespitzten Situation als »Warn- und Kampfschrift« geschrieben. Borkenau appellierte an die liberalen Demokratien, sich auf die zu erwartende militärische Konfrontation vorzubereiten. Denn:

This European war is an »ideological war.« It is a fight of the liberal powers against the biggest »totalitarian« power, Germany. And Germany, in this war, is cooperating, though in an ambiguous manner, with Russia, the other big totalitarian power of the world [...] the main devision could not be more clear-cut; liberal powers here, totalitarian powers there. ${ }^{164}$

Angesichts des Hitler-Stalin-Paktes zeigte sich für Borkenau vor allem beim Nationalsozialismus der neuartige Systemcharakter. Das »Neue« am deutschen Totalitarismus zeigte sich für Borkenau - vor dem Hintergrund der Erfahrungen, die die Weltöffentlichkeit mit den Methoden Hitlers z. B. beim Münchner Abkommen im September 1938 machte -, dass Hitler einen Bruch mit den Gepflogenheiten der westlichen Diplomatie vollzog, und zwar insofern, als er sich an keine Absprachen und Verträge hielt, seine Gegner unter Androhung von Gewalt einschüchterte, die demokratischen Staaten untereinander ausspielte und ihre Schwächen bedenkenlos ausnutzte. Für Borkenau bestand die Gefahr, die von der totalitären Außenpolitik Hitlers ausging, darin, dass er sich bei seiner unbegrenzten Expansionspolitik, d.h. seinem Ziel, Deutschland nach der Eroberung der östlichen Länder zur einzigen Weltmacht Nummer eins zu machen, durch keinerlei diplomatische Prinzipien binden lassen wollte. Auch aus diesen Gründen appellierte Borkenau für einen entschlossenen und einheitlichen militärischen Kampf der alliierten Streitkräfte. Die besondere Gefahr Hitlers bestand darin, dass er zu jedem Zeitpunkt mit jedem Staat entsprechende diplomatische Übereinkünfte eingehen würde, solange es seinen politischen Interessen diente. ${ }^{165}$

Der Aufstieg des Nationalsozialismus und des sowjetischen Kommunismus erfolgte für Borkenau aufgrund einer in der Folge des Ersten Weltkrieges entstandenen allgemeinen internationalen Krise, mit der die an den Schalthebeln der Macht sich befindende traditionelle politische Klasse keine eigenständige Lösung parat hatte. Bei allen Unterschieden in Bezug auf den gesellschaftsökonomischen Entwicklungsstand in den beiden Ländern stellte der Autor fest:

che moderner Cewaltregime liegen. Denn Voigt benutzte neben dem gelegentlich verwandten Totalitarismusbegriff vor allem das Konzept der Säkularreligion, das für ihn zu einer Art Schlüssel für die phänomenologische Erfassung der Cleichförmigkeiten von Marxismus und Nationalsozialismus wurde.« Zum Einfluss von F. A. Voigt auf The Totalitarian Enemy von Franz Borkenau siehe die Ausführungen von Markus Huttner, in: Ebd., S. 225-230. Vgl. auch Franz Borkenau, Rezension von F. A. Voigt, Unto Caesar, in: The Sociological Review 30 (1938), S. 421-424.

163 Borkenau, The Totalitarian Enemy, S. 13.

164 Ebd., S. 11.

165 Ebd., S. 172. 
Neither the Russian nor the German Revolution originated primarily in specific national conditions. Both were results of an international social, political, and economic crisis, and of one and the same crisis, to wit, that crisis which has held the world in its grip since $1914 .^{166}$

Der unterschiedliche gesellschaftsökonomische Entwicklungsstand in Deutschland und Russland hatte zur Folge, dass nach Borkenaus Auffassung die soziale Revolution in den beiden Ländern von unterschiedlicher Qualität war. Für ihn brauchte der Nationalsozialismus keine totalen Veränderungen im gesellschaftlichen Gefüge vorzunehmen, und mithin konnte die alte politische und wirtschaftliche Funktionselite übernommen werden. Ganz anders in Russland, wo die bolschewistische Machtergreifung einen gewissermaßen totalitären Austausch der gesamten alten Führungsschicht zur Folge hatte. ${ }^{167}$

Borkenaus Theorie zufolge waren also der Nationalsozialismus und der Sowjetkommunismus keineswegs »vom Himmel gefallen« (Arendt), sondern entstanden auf dem Boden einer inneren Krise der westlichen bzw. europäischen Gesellschaftsformation. ${ }^{168}$ Anders gesagt: Der Totalitarismus entsprang Borkenau zufolge einer Krise der Demokratien und der kapitalistischen Gesellschaft, sodass der Exkommunist und geschulte marxistische Theoretiker in dem Kampf gegen die totalitären Systeme die Notwendigkeit sah, in den demokratischen liberalen Staaten Eingriffe in den wirtschaftlichen Kreislauf vorzunehmen. Borkenau plädierte für staatliche Eingriffe in die Wirtschaft, die seiner Auffassung auch die in den liberalen Staaten existierenden gesellschaftlichen Probleme bewältigen könnten und mithin die Gefahr einer Re-

166 Ebd., S. 211.

167 Ebd., S. 235 f. Gemeinhin wurde zum Zeitpunkt der Veröffentlichung im Jahre 1940 der italienische Faschismus beim Vergleich mit dem Nationalsozialismus und dem Sowjetkommunismus noch unter die Kategorie des Totalitarismus subsumiert. Franz Borkenau war einer der ersten Totalitarismustheoretiker, der die qualitativen Differenzen der drei Herrschaftsregime herausstrich. Für ihn war der italienische Faschismus kein totalitäres System, weil »der Duce« Benito Mussolini darauf verzichtete, eine totale gesellschaftliche und politische Revolution vorzunehmen, d. h., keinen totalen Wechsel der alten Funktionseliten vornahm und im Großen und Ganzen auf staatliche Eingriffe verzichtete (S. 229).

168 Nach dem Tod seines Freundes schrieb Richard Löwenthal in seiner Einführung zu Franz Borkenau, Ende und Anfang. Von den Cenerationen der Hochkulturen und von der Entstehung des Abendlandes (Stuttgart 1984) auf S. 18, dass es zu den bemerkenswerten Leistungen Borkenaus zählte, in seiner Totalitarismusstudie erkannt zu haben, »daß die totalitären Ideologien unserer Zeit nicht die Produkte eines diabolus ex machina sind, der plötzlich den Westen von außen her bedroht, sondern einer inneren Krise der westlichen Zivilisation entsprungen sind. Der Nationalsozialismus war eine >Revolte gegen den Westen a aus seinem eigenen Bereich; und während die Kulturkrise, die ihn hervorrief, damals nur Deutschland erfaßte, hat sie nach Borkenaus Lebenszeit auch andere, weltweite Erscheinungsformen hervorgebracht. Der Bolschewismus kann historisch nur als das Resultat der Ausbreitung der westlichen Häresie des Marxismus auf dem nicht-westlichen Boden Rußlands verstanden werden. Die Drohung des stotalitären Feindes serschien so in Borkenaus Sicht als ein Zeichen, wie weit die inneren Faktoren kulturellen Zerfalls im Westen bereits fortgeschritten waren; und während er zur Zeit der Abfassung des Buches noch überzeugt war, daß die unmittelbare Gefahr durch einen Sieg des Westens überwunden werden könne, wurde er nach dem Zusammenbruch Frankreichs tief pessimistisch über den Kriegsausgang - und war bald nach dem Siege abermals pessimistisch für die Aussichten, einen neuen Weltkrieg ohne eine totale Kapitulation des Westens vor Stalins Sowjetunion zu vermeiden.« 
volution und von möglichen totalitären Tendenzen in den demokratischen Ländern abwenden könnten. ${ }^{169}$

Trotz der zweifelsohne totalitarismustheoretischen Ausrichtung von The Totalitarian Enemy lag der Schwerpunkt eindeutig auf der Untersuchung des Nationalsozialismus, dem der Autor sich in fünf umfangreichen Kapiteln zuwandte, ${ }^{170}$ sodass ein konzeptionelles Problem vorherrschte. Der profilierte Experte für den Kommunismus thematisierte in einem auffallend kleineren Abschnitt das sowjetische System. In der Untersuchung von Borkenau entstand dadurch das Problem, dass zum einen ebenjenes inhaltliche Ungleichgewicht vorherrschte, da die Auseinandersetzung mit dem deutschen Totalitarismus dominierte. Zum anderen verwischte der Autor objektive Systemunterschiede zwischen dem "Hitler-Regime« und dem "Stalin-Regime«, sodass bestehende Differenzen zugunsten einer "geschlossenen Theorie« zum Teil nicht berücksichtigt wurden. ${ }^{171}$

Auf der Basis der in den beiden Untersuchungen Austria and After ${ }^{172}$ und vor allem The New German Empire ${ }^{173}$ gewonnenen Erkenntnisse analysierte Borkenau den Nationalsozialismus unter Hitler. Neben den oben erwähnten allgemeinen Entstehungsursachen für das Phänomen Totalitarismus existierten zudem für Borkenau genuin länderspezifische Bedingungen. Das bedeutete für das >Dritte Reich $\measuredangle$ Im Vergleich $\mathrm{zu}$ anderen europäischen Ländern entstand in Deutschland nach dem verlorenen Weltkrieg eine gesellschaftliche und politische Krisensituation, auf der der Antiliberalismus, Antidemokratismus, Antiparlamentarismus, Antimarxismus, Antikonservatismus, Antisemitismus sowie die manifeste Kirchenfeindlichkeit der nationalsozialistischen Idee und Politik auf fruchtbaren Boden stoßen konnte. Allerdings konnte letzten Endes nach Auffassung von Borkenau die »Revolution des Nihilismus« (Rauschning) bzw. Hitlers Vorstellung einer revolutionären Bewegung des Nihilismus nur reüssieren, d. h. an die Macht kommen, weil die hitlersche Propaganda die auch durch die wirtschaftliche Situation in Deutschland entstandene "geistige « und soziale Krise in ihrem Sinne auszubeuten verstand. Das was später Arendt in ihrer Totalitarismusstudie als Bündnis zwischen »Mob und Elite «bezeichnete, konstatierte auch Borkenau. Nämlich, dass die Entwicklung der kapitalistischen Gesellschaft zur Auflösung der Klassen führte, sodass das nationalsozialistische Programm alle Indi-

169 Borkenau, The Totalitarian Enemy, S. 104.

170 Die einzelnen Kapitelüberschriften lauteten: »Nazi Economics«, »Towards Collectivism«, »Nazi Mentality and its Background«, »The New Tyranny« und»The Nazi War«.

171 An dieser Stelle sei noch mal daran erinnert, dass die Auseinandersetzung mit dem vergleichenden Totalitarismusbegriff-bereits in den späteren 1930er-Jahren, aber auch danach - im Wesentlichen von den außenpolitischen Konstellationen abhing. Das heißt in Bezug auf das Borkenau-Buch: Zum Zeitpunkt der Fertigstellung der Untersuchung stellte sich zwar den meisten Zeitgenossen das NSSystem im Vergleich zur Sowjetunion als das politisch und militärisch weitaus gefährlichere dar; daraus resultierte sicherlich die Tatsache, dass Borkenau sich in erster Linie mit dem NS-System beschäftigte. Da jedoch angesichts des Hitler-Stalin-Paktes die beiden totalitären Mächte den liberalen Demokratien als »gleich « gefährliche Gegner erscheinen sollten, flossen bestehende Differenzen nicht in den identifizierenden Totalitarismusbegriff hinein.

172 Franz Borkenau, Austria and After, London 1938.

173 Ders., The New German Empire, Harmondsworth 1939. 
viduen der »atomisierten Massen« (Arendt) ansprechen konnte. ${ }^{174}$ Der messianische Dimensionen annehmende Glaube an Hitler in der deutschen Bevölkerung, den die NS-Propaganda geschickt ausnutzte, resultierte auch in der Hoffnung auf eine ökonomische Erneuerung bzw. staatliche Wirtschaftslenkung Deutschlands. Worum es dem NS-Regime allerdings in den Augen von Borkenau ausschließlich ging, war der Erhalt der politischen Macht. ${ }^{175}$

Das Ziel des Machterhalts korrespondierte, so Borkenau, mit außenpolitischen Ambitionen des Nationalsozialismus, sodass Hitler einen Konnex zwischen Wirtschaftspolitik und Wiederaufrüstungsprogramm herstellen konnte. ${ }^{176}$ Denn nur mit dem Abbau der Arbeitslosigkeit und der militärischen Wiederaufrüstung Deutschlands waren die letzten Endes unbegrenzten außenpolitischen Eroberungs- und Herrschaftsziele zu realisieren. Und dieses zentrale Ziel bestand in dem hitlerschen Plan der Weltherrschaft. Dafür wurde fast der gesamte wirtschaftliche Bereich vom NSStaat okkupiert und im Hinblick auf eine Kriegswirtschaft ausgerichtet, sodass quasi ein staatlicher »Rüstungskapitalismus « entstand. ${ }^{177}$ Die geplanten sozialen Folgekosten für die Bevölkerung, wie z. B. erhöhte Arbeitszeiten und schlechtere Ernährung, wurde von der nationalsozialistischen Führung mit dem Mittel der »messianischen Hoffnungspropaganda « ${ }^{178}$ verkauft. Denn ein Stillstand der nationalsozialistischen »Revolution « durfte nicht eintreten. ${ }^{179}$

Die nationalsozialistische Ideologie wurzelte für Borkenau in einer in der deutschen (Geistes-)Geschichte angelegten »Revolte gegen den Westen« (Löwenthal), die sich erfolgreich in den Jahren nach dem verlorenen Ersten Weltkrieg in der Bevölkerung Bahn brechen konnte. Die wichtigsten Elemente waren dabei »die Betonung einer nationalen Einheit, verbunden mit einer Heils- und Erlösungslehre, deren pseudowissenschaftlicher Fundierung und die Propagierung eines umfassenden Feindbildes ${ }^{180}$. Das heißt, neben der destruktiven Rolle des utopischen deutschen Nationalismus, der

174 Borkenau nahm sehr wohl zu Kenntnis, dass Hitler, nachdem er sich für die »legale Revolution « entschieden hatte, bewusst das Bündnis mit Gegnern der Weimarer Republik in den Reihen der konservativen Elite gesucht hatte, um das verhasste »System« zum Einsturz zu bringen und höchstselbst an die Macht zu kommen, um danach mit den linken und rechten Gegnern abzurechnen. In The New German Empire schrieb Borkenau: »lt is therefore meaningless to ask whether Nazi-ism is a Socialist revolution against the old ruling classes or a reaction of the upper classes against the threat from the masses below. With the advent of Nazi-ism, a political body recruited from all classes but independent of them all has taken power in its hands and established its absolute domination.«(Ebd., S. $20 \mathrm{f}$.)

175 Borkenau, The Totalitarian Enemy, S. 32.

176 Ebd., S. 32-37.

177 Ebd., S. 38-50.

178 Birgit Lange-Enzmann, Franz Borkenau als politischer Denker, Berlin 1996, S. 172.

179 Die Autorin der ausgezeichneten Borkenau-Werkbiografie, Birgit Lange-Enzmann, weist zu Recht in ihrer Untersuchung (ebd., S. 172) auf die offensichtlichen Parallelen zu dem »Klassiker der Totalitarismusforschung« (Alfons Söllner), der 1942 veröffentlichten Studie Permant Revolution. The Total State in a World at War von Sigmund Neumann hin. Siehe meine Ausführungen zur Studie von Sigmund Neumann in dem Kap. I. Zur grundsätzlichen Bedeutung von Neumanns Buch und zu dessen Einfluss auf die Totalitarismusstudien von Hannah Arendt und Carl ]. Friedrich vgl. Söllner, Sigmund Neumanns Permanent Revolution, in: Söllner/Walkenhaus/Wieland, Totalitarismus, S. 53-65. 
rassistischen Theorie der deutschen Herrenrasse und dem mit messianischen Zügen ausgestatteten Führer Adolf Hitler sah Borkenau in dem Feindbild des Juden ein überragendes Element der nationalsozialistischen Weltanschauung. Die Juden firmierten - so könnte man Borkenau verstehen - als »Gegenrasse« und gewissermaßen als »natürlicher Feind« Deutschlands, da sie den »deutschen Werten« antithetisch gegenüberstünden. "If in the Nazi faith the Jew is the arch-enemy, then the Nazi methods of Jew-baiting must be regarded as the incarnation of the Nazi spirit. And that spirit is one of sadistic torture of helpless and innocent victims. ${ }^{181}$

Die »Nazielite« um Göring, Goebbels, Röhm und Streicher unter der Führung Hitlers hatten als »Psychopathen« und »Politkriminelle« ein instrumentelles Verhältnis zur nationalsozialistischen Ideologie. Nach Borkenau waren die Exponenten dieser Führungselite weniger Überzeugungspolitiker bzw. -täter, d.h., sie glaubten selber nicht an ihre vertretene Ideologie, sondern sahen in ihr in allererster Linie ein Instrument $^{182}$ für den politischen Kitt zwischen dem Herrschaftsregime im engeren Sinne und der deutschen Bevölkerung und mithin zur »Machtsicherung der Elite«. Gleichwohl bestand Borkenau auf einer Trennung zwischen der nationalsozialistischen Führung und Ideologie auf der einen Seite sowie der deutschen Bevölkerung auf der anderen Seite. In Bezug auf die Rolle Deutschlands nach dem Krieg äußerte er sich über die Deutschen in The Totalitarian Enemy folgendermaßen: »Then there is no reason whatsoever why her legitimate claims should not be fulfilled. But if she were likely to remain a centre of disturbance no other policy would be open but to prevent her from being dangerous. $\aleph^{183}$

In einem eher knapp bemessenen Abschnitt seiner Studie thematisierte Borkenau neben den Entstehungsbedingungen die Verlaufsform der bolschewistischen Revolution, die schlussendlich zum totalitären Herrschaftsregime unter Stalin führte, und stellte hier ausdrücklich Vergleiche zum Nationalsozialismus an. Wie nach ihm Carl J. Friedrich ${ }^{184}$ betrachtete Borkenau die Einführung des ersten Fünfjahrespla-

181 Borkenau, The Totalitarian Enemy, S. 138.

182 Im Gegensatz zu Franz Borkenau inistierte Hermann Rauschning darauf, dass der Antisemitismus beim nationalsozialistischen »Führer « und deutschem Staatsoberhaupt keineswegs nur instrumentellen Charakter hatte und mithin vor allem propagandistische Zwecke erfüllte. Rauschning beschrieb Hitler als einen ideologischen Antisemiten, der von seinem »Glauben « an den existentiellen »Entscheidungskampf« gegen die Juden überzeugt war, siehe hierzu Rauschning, Gespräche mit Hitler, S. 220-228.

183 Borkenau, The Totalitarian Enemy, S. 145. Die von Borkenau zum Zeitpunkt der Buchveröffentlichung 1940 - also vor den eigentlichen Verbrechen der Deutschen, der Vernichtung der europäischen Juden - vorgenommene Trennung zwischen Herrschaftsregime im engen Sinn und deutscher Bevölkerung sollte bekanntlich hinfort für die Redaktion in der Zeitschrift Der Monat um Melvin ]. Lasky vorherrschend sein, wenn es einerseits um die Analyse des Nationalsozialismus ging und andererseits um die des Sowjetkommunismus unter Stalin. Anders gesagt: die Trennung von »Regime und Volk« war nachgerade die analytische Prämisse, wenn Der Monat den theoretischen Fokus auf die totalitären Herrschaftsregime in Deutschland und der Sowjetunion richtete.

184 In seinem totalitarismustheoretischen Standardwerk schrieb Carl ]. Friedrich: »Den entscheidenden Anstoß für einen zentralen Staatsplan gab die politische Entscheidung, das Land in großem Umfang zu industrialisieren und die Landwirtschaft zu kollektivieren. Erst damit beginnt im Jahre 1928 die Ära der Fünfjahrespläne. Seither dreht sich das Wirtschaftsleben der Sowjetunion um diese breit angelegten, umfassenden Pläne, wie sie die Planer der Cosplan-Organisation auf Grund der grundsätzlichen Entscheidung der Führung entwickelt haben. Ja, man darf vielleicht sagen, daß die Inan- 
nes als entscheidende historische Zäsur des sowjetischen Staates, der eine politische Grundsatzentscheidung Stalins vorausging. Mit der Beseitigung jedweder privatwirtschaftlicher Aktivitäten der Kulaken und der Einführung der Kollektivierungspolitik des sowjetischen Staates ging es für Borkenau um die Herrschaftssicherung der kommunistischen Partei und ihres »Führers« Stalin. Dessen Kollektivierungspolitik mit Millionen von Toten unter den Kulaken und der in diesen Kreisen einsetzende Widerstand hatten nach Einschätzung Borkenaus zur Folge, dass der »totalitäre Führer« eine Terror- und Unterdrückungspolitik in Gang setzte, von der die Bevölkerung, die politische und militärische Klasse des Landes, betroffen war. Die Opfer der stalinschen Terrorpolitik mussten als Sündenböcke einer mit katastrophalen Folgen verbundenen staatlichen Planwirtschaft herhalten. Anders gesagt: Die permanente Suche nach Schuldigen einer verfehlten (Wirtschafts-)Politik war dem kommunistischen System unter Stalin quasi immanent. Ohne die Existenz eines »inneren Feindes« konnte der sowjetische Staat der Ära Stalins nicht existieren. Aus diesem Grund war für Borkenau die Sowjetunion im Vergleich zu Deutschland die eigentliche Inkarnation des Totalitarismus. Denn, so Borkenau: »Russia is the totalitarian country par excellence; Communism the purest and most logical form of totalitarianism «. ${ }^{185}$ Darüber hinaus kam Borkenau zu der Einsicht, dass für ein totalitäres Herrschaftssystem die Notwendigkeit der Existenz eines Feindbildes absolut notwendig ist. ${ }^{186}$

Die für das totalitäre Herrschaftssystem implizite Feindobsession, das für die Machthaber notwendige Vorhandensein eines »existentiellen Gegners«, drückte sich für Borkenau auch durch die Tatsache aus, dass sie die eigene Einparteienherrschaft und den eigenen Staat umgeben sahen von »äußeren Feinden«, mit denen man qua definitiom nicht in Frieden leben könne. Dazu Borkenau:

The existence of other political regimes abroad is an unbearable barrier to any totalitarian règime. $A$ liberal and democratic régime can easily put up with national differences. A totalitarian regime cannot. Its Führer is the super-man; its changing tenets are the truth for the day when are uttered. Every disagreement is a crime. Nazism cannot have the full validity it claims as long as people next door are living happily without it. The same applies in a still higher degree to Communism. ${ }^{187}$

Nur ein Jahr nach der Veröffentlichung von Borkenau war es der trotzkistische Renegat James Burnham, der mit The Managerial Revolution eine totalitarismustheoretische

griffnahme des ersten Fünfjahresplanes als Anfang der eigentlich totalitären Revolution angesehen werden kann. Stalins Programm, das im wesentlichen mit den Forderungen der sogenannten linken Opposition übereinstimmte, forderte natürlich den Widerstand der Bauern und anderer Cruppen heraus. Man begegnete ihm mit einer Verschärfung der Zwangsmaßnahmen. Da jede Gesellschaft ein kompliziertes System von sich überschneidenden Interessenzusammenhängen ist, so ergriff bald die totalitäre Revolution die gesamte Wirtschaft.« (Friedrich, Totalitäre Diktatur, S. 175 f.)

185 Borkenau, The Totalitarian Enemy, S. 229.

186 Ebd., S. 227.

187 Ebd., S. $232 \mathrm{f}$. 
Untersuchung vorlegte, ${ }^{188}$ die zeitweilig eine sehr populäre Theorie darstellte. ${ }^{189}$ Die in der Totalitarismusstudie entfalteten umstrittenen Ansichten des späteren Neo-Machiavellisten ${ }^{190}$ implizierten erste Ansätze einer Konvergenztheorie, ${ }^{191}$ da für Burnham das »Managerregime« sich nicht nur in Deutschland und der Sowjetunion an der Macht befand, sondern auch in den Vereinigten Staaten totalitäre Angleichungstendenzen davon zu konstatieren waren. »Der New Deal«, so der Autor,

ist weder Stalinismus noch Nazismus. Er ist nicht einmal eine amerikanische Analogie dazu; denn der New Deal ist in seiner manageriellen Entwicklung viel weiter zurück, und der Kapitalismus ist in den Vereinigten Staaten noch nicht vorüber. Aber ein ehrlicher Beobachter, sei er nun Freund oder Feind des New Deal, kann nicht leugnen, daß der New Deal hinsichtlich der wirtschaftlichen, sozialen, politischen und ideologischen Abkehr vom traditionellen Kapitalismus die gleiche Richtung verfolgt wie Stalinismus und Nazismus. Der New Deal ist eine Phase des Übergangs vom Kapitalismus zur Managergesellschaft. ${ }^{192}$

188 James Burnham, The Managerial Revolution, New York 1941 (dt. Ausg.: Das Regime der Manager, Stuttgart 1948). James Burnham wurde als Sohn englischer Einwanderer 1905 in Chicago geboren (gestorben 1987 in Kent/Connecticut). Er studierte Philosophie, Volkswirtschaft und Staatswissenschaft an der Universität in Princeton und am Balliol College in Oxford. Zu Beginn des Jahres 1930 nahm er eine Lehrtätigkeit an der philosophischen Abteilung des Washingtoner Square College an der New Yorker Universität auf und hatte dort einen Lehrstuhl von 1932 bis 1954. Von 1930 bis 1933 gab er die Vierteljahreszeitschrift The Symposion heraus. Zu diesem Zeitpunkt dominierte bei Burnham die Sozialkritik am kapitalistischen Gesellschaftssystem. Ab 1933 avancierte er zum Anhänger des Trotzkismus und wurde zum starken Kritiker des kommunistischen Systems stalinscher Couleur. Burnham brachte einige Jahre die antistalinistische Zeitschrift The New International heraus. Nach überaus heftiger Kontroverse brach Burnham mit dem im mexikanischen Exil lebenden Trotzki, weil es unterschiedliche theoretische und politische Auffassungen über die Bedeutung der Russischen Revolution gab. In den folgenden Jahren war Burnham Mitherausgeber der amerikanischen Zeitschrift Partisan Review, die, wie bereits gesagt wurde, zu einem früheren Zeitpunkt trotzkistisch ausgerichtet war und ab Ende der 1930er-Jahre eine militant antikommunistische Ausrichtung besaß. Nach der Veröffentlichung seiner Totalitarismusstudie veröffentlichte Burnham The Struggle for the World (1947) und The Coming Defeat of Communism (1950), mit denen er in der Hochphase des Kalten Krieges für internationales Aufsehen sorgte. Neben der Analyse und radikalen Kritik des sowjetischen Kommunismus unter Stalin trat Burnham darin für ein vehementes und offensives Verteidigen der »westlichen Werte« ein. Später war Burnham Herausgeber des Zentralorgans der amerikanischen Konservativen, der Zeitschrift National Review. Siehe einzelne biografische Angaben zu Burnham in Wald, The New York Intellectuals, S. 175-182.

189 Jänicke, Totalitäre Herrschaft, S. 113.

190 James Burnham, Die Machiavellisten. Verteidiger der Freiheit, München 1949.

191 Mit der komparativ ausgerichteten Konvergenztheorie beschäftigte sich die Wissenschaft systematischer zum Zeitpunkt der beginnenden Ost-West-Entspannungspolitik in den 1960er-Jahren. Aus der umfangreichen Forschungsliteratur vgl. Reinhard Bendix, Herrschaft und Industriearbeit. Untersuchungen über Liberalismus und Autokratie in der Ceschichte der Industrialisierung, Frankfurt a. M. 1960; Ceorge Paloczi-Horvath, Rebellion der Tatsachen. Die Zukunft Rußlands und des Westens, Frankfurt a. M. 1963; Zbigniew K. Brzezinski/Samuel P. Huntington, Political Power: USA/ USSR, London 1964 (dt. Ausg.: Politische Macht. USA/UdSSR, Köln/Berlin 1966); Ernst Richert, Die neue Cesellschaft in Ost und West, Analyse einer lautlosen Revolution, Cütersloh 1966.

192 Burnham, Das Regime der Manager, S. 299 f. 
Anders gesagt: Burnham gelangte zur Feststellung, dass der Kapitalismus nicht mehr vorrangig von den Kapitalisten getragen wurde. Seit dem Ersten Weltkrieg habe sich an ihrer Statt ein (weltweites) »Regime der Manager« über Staat und Wirtschaft etabliert. Hierbei handelte es sich um einen Funktionärstypus, der mit der Gesellschaft so umginge wie mit einer Fabrik. Dieser Personenkreis, den der Autor unter den Begriff »Manager« subsumierte, setzte sich aus Industriellen, Technikern, Parteibürokraten und Armeeoffizieren zusammen und war nicht mehr primär am Profitmotiv, sondern in erster Linie am Machtmotiv interessiert. ${ }^{193}$ Die Managerelite war ausschließlich an der politischen Macht interessiert und hierbei hatten in Bezug auf den Nationalsozialismus und den Sowjetkommunismus neben dem »Massenbewegungscharakter", der »Einparteiendiktatur«, dem »Führerprinzip« die »Ideologien der Manager« eine zentrale Bedeutung. ${ }^{194}$ In Russland hatte sich nach Einschätzung von Burnham der erste Managerstaat in der Geschichte etabliert. ${ }^{195}$

Für Burnham besaß die für die marxistische Theorie entscheidende Frage nach dem Eigentum an den Produktionsmitteln in Bezug auf den Totalitarismus keine Bedeutung mehr. Wie der Verlauf der Russischen Revolution gezeigt hatte, führte die Abschaffung des kapitalistischen Privateigentums nicht - wie es die marxistische Revolutionstheorie voraussagte - zwingend zum Sozialismus, d.h. einer freien und klassenlosen Gesellschaft. ${ }^{196} \mathrm{Ganz}$ im Gegenteil führte die Revolution zu einer neuen Klassen- bzw. Managergesellschaft. Und der sowjetische Staat war für Burnham ein totalitärer Staat, der vom selben Typ war, wie er sich nach der nationalsozialistischen »Revolution« in Deutschland entwickelt hatte. Für Burnham war es zweifelsohne richtig, dass die

Herrschaft einer privilegierten Klasse deren Kontrolle über die Produktionsmittel voraussetzt; aber die Kontrolle braucht nicht durch individuelle Eigentumsrechte ausgeübt zu werden. Das kann vielmehr auch durch sogenannte korporative Rechte geschehen, die im Besitz nicht von Privatpersonen, sondern von Institutionen sind. So war es z. B. in vielen Gesellschaften mit einer herrschenden Priesterkaste, also bei zahlreichen

193 So Neumann, Behemoth, S. 273. In seinem zum Standardwerk der NS-Forschung avancierten Buch setzte sich Neumann kritisch mit Burnhams These auseinander, dass auch der Nationalsozialismus seinen kapitalistischen Charakter verloren habe. Neumann dagegen ging bei seiner Analyse des Nationalsozialismus noch vom Primat der Ökonomie über die Politik aus. Später vertrat Neumann eine andere Auffassung. In seinem 1955 geschriebenen Aufsatz Ökonomie und Politik im zwanzigsten Jahrhundert sprach Neumann in Bezug auf den Nationalsozialismus von der »Suprematie der Politik über die Ökonomie«. Franz Neumann, Ökonomie und Politik im zwanzigsten Jahrhundert, in: Ders., Demokratischer und autoritärer Staat. Beiträge zur Soziologie der Politik, Frankfurt a. M. 1967, S. 171-183, hier S. 180. Interessanterweise kam bereits 1939 Richard Kern (d. i. der frühere marxistische Ökonomietheoretiker und sozialdemokratische Politiker Rudolf Hilferding) in einem Artikel im Neuen Vorwärts mit Blick auf Deutschland, Sowjetrußland, Italien (und Japan) zu der Feststellung, dass in diesen Ländern der Primat der Politik über die Wirtschaft galt. Für diese sogenannten totalitären Gesellschaften zählte die Tatsache, dass die wirtschaftlichen Ziele den politischen Zielen untergeordnet waren. Vgl. Rudolf Hilferding, The Modern Totalitarian State, in: Modern Review 1 (1947), S. 597-605.

194 Siehe Burnham, Das Regime der Manager, S. 219-242.

195 Ebd., S. 247.

196 Vgl. hierzu auch Koestler, Der Yogi und der Kommissar, S. 328-334, der sich hier ausdrücklich auf die Studie von Burnham berief. 
primitiven Kulturen, im alten Ägypten und in gewissem Umfang auch im Mittelalter. In solchen Gesellschaften kann es wenige Reiche und viele Arme, wenige Mächtige und viele Unterdrückte geben, genau wie das (z. B. im Kapitalismus) bei Cesellschaften der Fall ist, in denen die Eigentumsrechte bei den Individuen als solchen liegen. ${ }^{197}$

Eingedenk der historischen Vorläufer kam Burnham angesichts seiner Erkenntnis, dass im "Regime der Manager Privatleute kein entsprechendes Eigentum besaßen und mit Blick auf die Frage, wie dann überhaupt ein Gruppe von Menschen eine herrschende Klasse bilden kann, zu folgender Antwort: »Nicht durch Eigentumsrechte, die sie als einzelne besitzen, werden die Manager die Kontrolle über die Produktionsmittel ausüben und bei der Verteilung bevorzugt werden, sondern mittelbar durch die Kontrolle über den Staat, der seinerseits die Produktionsmittel zu Eigentum besitzt und kontrolliert. $^{198}$

Der in der Studie The Managerial Revolution von Burnham entfaltete Kerngedanke der Konstituierung des oligarchischen Kollektivismus anstatt einer sozialistischen Gesellschaft stieß bei Koestler, Borkenau ${ }^{199}$ als auch - wie erwähnt - bei Orwell auf großes Interesse. Insbesondere hatte die burnhamsche Prognose, dass hinfort die Welt von drei großen Superstaaten beherrscht werden, die untereinander in einem permanenten Kriegszustand leben werden, vor allem für das totalitarismuskritische Buch Nineteen Eighty-Four Orwells eine außerordentliche Bedeutung. ${ }^{200}$

\subsection{Zur Bedeutung der Renegaten für die Totalitarismustheorie}

Mit The Totalitarian Enemy von Borkenau und The Managerial Revolution Burnhams lagen zu einem frühen Zeitpunkt totalitarismustheoretische Studien von zwei ehemaligen Kommunisten respektive Trotzkisten vor. Insbesondere die Studie von Borkenau avancierte zu einem Standardwerk der Totalitarismusforschung. Darüber hinaus

197 Burnham, Das Regime der Manager, S. $56 \mathrm{f}$.

198 Ebd., S. 92.

199 Siehe Franz Borkenau, Die Revolution der Manager, in: Merkur 3 (1949), H. 5, S. 487-494.

200 Ceorge Orwell schrieb in dem Aufsatz James Burnham and the Managerial Revolution aus dem Frühjahr 1946 zustimmend: »Die neuen >Manager-Gesellschaften werden nicht aus einem Flickenteppich kleiner, unabhängiger Staaten, sondern aus großen Superstaaten bestehen, die sich um die industriellen Hauptzentren in Europa, Asien und Amerika konzentrieren. Diese Superstaaten werden untereinander um den Besitz der noch nicht mit Beschlag belegten Teile der Welt kämpfen, aber sie werden wahrscheinlich nicht in der Lage sein, einander völlig zu besiegen. Im Innern wird jede Gesellschaft hierarchisch sein mit einer Begabtenaristokratie an der Spitze und einer Masse von Halbsklaven an der Basis.« (Zit. n. Hans-Christoph Schröder, George Orwell. Eine intellektuelle Biographie, München 1988, S. 193) - Kritisch äußerte sich Orwell hinsichtlich Burnhams Vorstellung, dass dieser die Stabilität der totalitären Herrschaftsregime in der Sowjetunion und in Deutschland überschätzt und die zweifelsohne vorhandenen systemimmanenten Zerstörungskräfte in ebendiesen Staaten mehr oder weniger unterschätzt. Mit Blick auf die Sowjetunion prognostizierte Orwell, dass sich das Regime unter Stalin entweder demokratisieren werde oder ganz verschwindet. Denn, so Orwell: »Das riesige, unbesiegbare, ewigwährende Sklavenreich, von dem Burnham zu träumen scheint, wird nicht errichtet werden, oder, wenn es errichtet wird, wird es nicht dauern, weil die Sklaverei nicht länger mehr eine stabile Grundlage für die menschliche Gesellschaft darstellt.« (Zit. n. ebd., S. 195) 